\title{
Hemangioma hepático congénito gigante: síndrome de Kasabach-Merritt
}

\section{Giant Congenital Liver Hemangioma: Kasabach Merritt Syndrome}

Carlos Cortés-Caballero, MD, ${ }^{1}$ Margarita Rosa Mogollón-Zehr, ${ }^{2}$ Silvia Nathalia Vera-Campos ${ }^{2}$

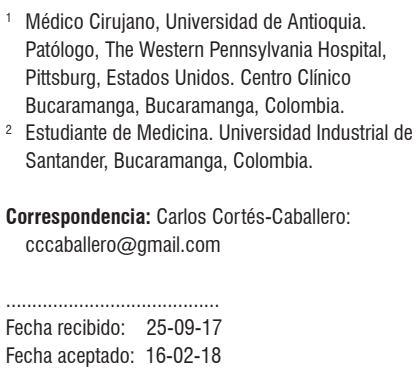

\begin{abstract}
Resumen
Los hemangiomas son las proliferaciones más frecuentes en la edad pediátrica, y el hemangioma hepático es de los más comunes entre los viscerales. El diagnóstico precoz es importante para orientar el tratamiento. Se presenta el caso de un neonato con compromiso ventilatorio secundario al efecto de una masa gigante, que ecográficamente correspondió a un hemangioma hepático. Se realizó resección quirúrgica y, ulteriormente, fue enviada a patología, que confirmó el diagnóstico.
\end{abstract}

\section{Palabras clave}

Hemangioma hepático, síndrome de Kasabach-Merritt, hemangioma gigante.

\begin{abstract}
Keywords

Hepatic hemangioma, Kasabach Merrit syndrome, giant hemangioma.
\end{abstract}

Hemangiomas are the most frequent proliferations in children, and liver hemangiomas are the most common among those in the viscera. Early diagnosis to focus treatment is important. We present the case of a neonate with compromised breathing secondary to the effect of a giant mass that echographically appeared to be a hepatic hemangioma. Surgical resection and subsequent pathology confirmed the diagnosis.

\section{INTRODUCCIÓN}

Los hemangiomas son las neoplasias hepáticas más frecuente en niños. Cerca del $70 \%$ se presentan al nacer y representan el $10 \%$ de los tumores pediátricos. Pueden comprometer cualquier órgano, aunque son más comunes en la piel, el hígado y el encéfalo (1). Su incidencia es mayor en mujeres, en una proporción de 3:1 (2).

Los hemangiomas se caracterizan por una proliferación de células endoteliales sin atipia y angiogénesis, lo que lleva a un rápido crecimiento de esta lesión. Por lo general, son de tamaño pequeño y asintomáticos; sin embargo, se han presentado casos de hemangiomas gigantes, definidos aquellos en los que su tamaño es mayor de $5 \mathrm{~cm}$ (3), que pueden generar una serie de complicaciones tales como: insuficiencia cardíaca, hipotiroidismo, síndrome de compartimento abdominal y síndrome de Kasabach-Merritt, que causa una alta mortalidad en neonatos.

Generalmente, su diagnóstico es incidental, mediante hallazgos ecográficos, aunque se puede confirmar histológicamente usando marcadores tipo transportador de glucosa 1 (GLUT1) (2), factor VIII, CD31 y CD38, lo que ayuda a su distinción de otras patologías vasculares (4).

\section{CASO CLÍNICO}

Paciente producto de un embarazo pretérmino de 33,3 semanas de gestación, de una mujer de 29 años con ante- 
cedentes de diabetes gestacional controlada y corioamnionitis. Se observa un neonato de sexo femenino, de $2565 \mathrm{~g}$ y $45 \mathrm{~cm}$ de talla, con bradicardia y compromiso ventilatorio secundario a restricción por masa. Ingresa a la unidad de cuidados intensivos (UCI), donde se le realiza intubación orotraqueal. Durante el examen físico se encuentra abdomen distendido y se palpa una masa de consistencia dura en el flanco y en el hipocondrio izquierdo sin dolor ni signos de irritación peritoneal. El perímetro abdominal es de $35 \mathrm{~cm}$, que para su edad cronológica está aumentado; presenta petequias, piel y conjuntivas ictéricas.

Se solicita ecografía abdominal que muestra masa de ecogenicidad heterogénea dependiente del lóbulo hepático izquierdo, de difícil cuantificación por su tamaño, la cual se extiende hasta la pelvis desplazando estructuras vecinas. Se acompaña de patrón de vascularización en su interior y áreas quísticas sin calcificaciones, con posibilidades diagnósticas de hemangioma hepático versus hamartoma mesenquimal y, menos probable, de hepatoblastoma. Se reporta radiografía de tórax que sugiere cardiomegalia.

$\mathrm{Al}$ tercer día se realizan exámenes paraclínicos, que informan una bilirrubina total de $8,9 \mathrm{mg} / \mathrm{dL}$ y una bilirrubina indirecta de 7,9 mg/dL; ALAT de $21 \mathrm{U} / \mathrm{L}$; ASAT de 213 $\mathrm{U} / \mathrm{L}$; tiempo de protrombina prolongado, plaquetas de $56000 / \mathrm{mm}^{3}$; hemoglobina $(\mathrm{Hb})$ de $10,8 \mathrm{~g} / \mathrm{dL}$; y hematocrito (Hto) del 34,4\%. La evolución muestra la disminución progresiva de estos valores. Se cuantifican los niveles de alfafetoproteína (AFP), que informan $1000 \mathrm{UI} / \mathrm{mL}$. Con la impresión diagnóstica por tomografía axial computarizada (TAC) del hemangioma hepático congénito, se decide realizar lobectomía izquierda.

El día 14 del preoperatorio, la bilirrubina total es de $0,7 \mathrm{mg} / \mathrm{dL}$ y la bilirrubina directa es de $0,56 \mathrm{mg} / \mathrm{dL}$, con valores de las pruebas de función hepática y tiempo de protrombina dentro de los rangos normales. A los 10 días posquirúrgicos, presenta hemorragia pulmonar masiva de difícil manejo, con diagnóstico de coagulación intravascular diseminada (CID), por lo que se inicia tratamiento de rescate con adrenalina, ácido tranexámico y transfusión de crioprecipitado y plaquetas, con evolución satisfactoria. Además, se encuentra una hiperbilirrubinemia a expensas de la bilirrubina directa, con valores de bilirrubina total de $17 \mathrm{mg} / \mathrm{dL}$ y de bilirrubina directa de $12 \mathrm{mg} / \mathrm{dL}$. Ulteriormente se remite a consulta de cardiología pediátrica por sospecha de conducto arterioso persistente. Se considera que no requiere tratamiento quirúrgico y se da salida a los 45 días, en condiciones óptimas.

Se envía pieza de resección a patología. En los hallazgos histopatológicos se encuentra masa ovoide, firme, que pesa $268 \mathrm{~g}$, mide $10,5 \times 6,5 \times 5 \mathrm{~cm}$, presenta una superficie externa lisa y brillante; la base de implantación es cruenta, y alcanza 4 x $3 \mathrm{~cm}$; al seccionarla, está compuesta por un tejido rojo central rodeado por una banda pálida de espesor variable, que alcanza los $0,3 \mathrm{~cm}$ (Figura 1).

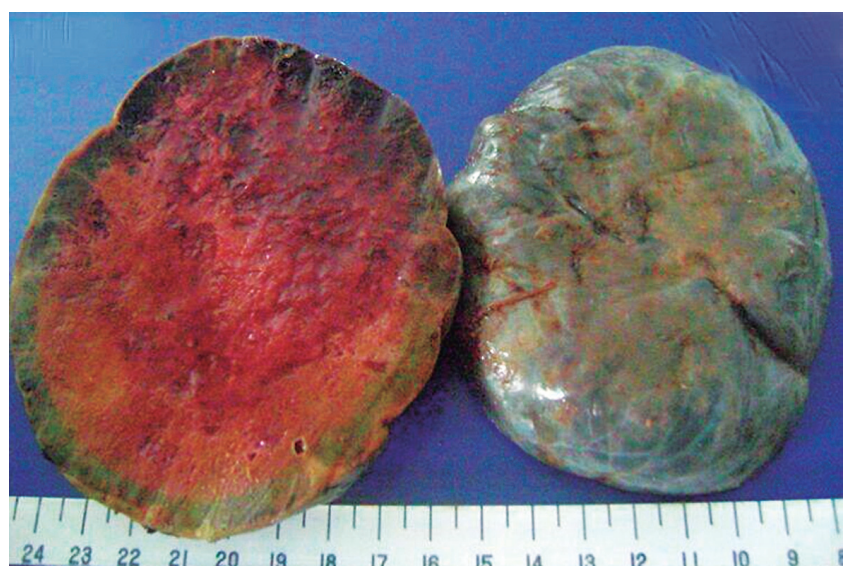

Figura 1. Hemangioma hepático con superficie externa lisa, compuesto por un tejido rojo central.

Microscópicamente predominan los espacios vasculares de diferente tamaño, algunos capilares y otros quísticos, tapizados por un endotelio monomórfico. La luz contiene glóbulos rojos e histiocitos con pigmento. En la parte periférica se observa una cápsula fibrosa, de espesor variable, con conductos biliares en algunos campos o con hepatocitos y capilares en otros. Se formula el diagnóstico de hemangioma hepático mixto capilar y cavernoso (Figura 2).

\section{DISCUSIÓN}

Los hemangiomas hepáticos son neoplasias benignas de origen mesenquimal y suelen ser solitarios. Según diversos estudios de dermatología y cirugía, se considera que el hemangioma presenta dos alteraciones: malformaciones vasculares y neoplasias vasculares (5). Estas crecen rápidamente en los primeros años de vida y se estabilizan aproximadamente a la edad de 1 año. Un $75 \%$ de los casos tiende a involucionar por completo hacia la edad de 5 a 7 años, por lo que su manejo se basa en el control de la evolución (6). En cuanto a la localización, se ha descrito con mayor frecuencia en el lóbulo hepático izquierdo (3), lo que concuerda con el presente caso.

Su etiología todavía sigue siendo desconocida, aunque existe una asociación entre el tamaño y los niveles de estrógeno/progesterona, con el crecimiento de la lesión observado durante el embarazo y el uso de anticonceptivos orales y de terapias de reemplazo hormonal (7) que, a su vez, pueden generar peliosis hepática (8). Esto explicaría la mayor prevalencia en el sexo femenino. En nuestro caso no se encontraron antecedentes familiares o de uso de estos fármacos; tampoco se halló relación con la diabe- 

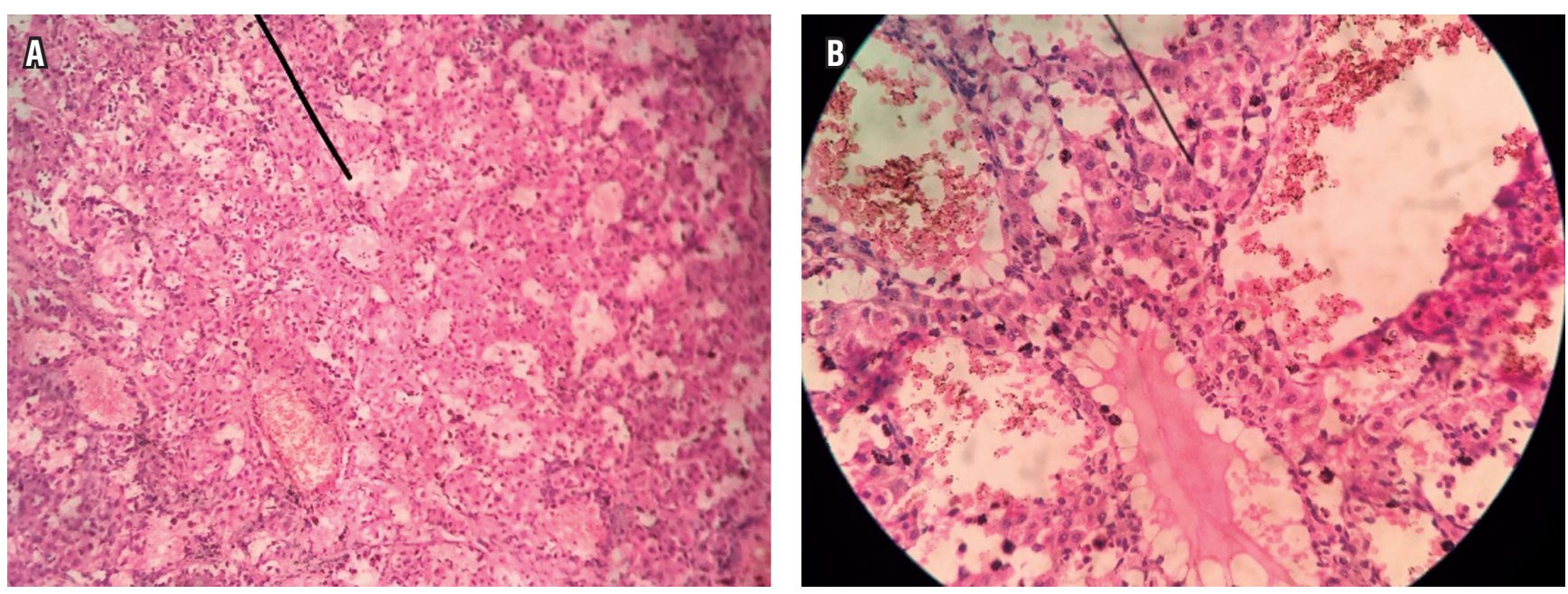

Figura 2. A. Estudio microscópico (HE, 20x) que muestra espacios vasculares tapizados por un endotelio sin atipia. B. (HE, 40x) glóbulos rojos en su interior e histiocitos con pigmento.

tes gestacional y la corioamnionitis que padeció la madre durante el embarazo.

Teniendo en cuenta que entre los diagnósticos diferenciales se encuentran los tumores abdominales propios de la infancia, se cuantificó la AFP con valores normales para la edad, por lo que la posibilidad de un hepatoblastoma es mínima. Este marcador debe interpretarse de acuerdo con el grupo etario y en los recién nacidos es muy elevado considerando este resultado normal para la edad.

En cuanto a las complicaciones, están la trombosis intratumoral, la ruptura, el diagnóstico incierto, la disfunción hepática o de órganos abdominales relacionada con el efecto de masa, el síndrome de coagulopatía, conocido como síndrome de Kasabach-Merritt, y la muerte, que en la mayoría de los casos se debe a insuficiencia cardíaca que se produce aproximadamente en el $50 \%$ de los pacientes con hemangioma hepático y es secundaria a la derivación arteriovenosa de lesiones de gran tamaño (6). En la paciente hubo hallazgos clínicos y de laboratorio sugestivos de anemia hemolítica y trombocitopenia severa acompañada de coagulopatía de consumo, hallazgos propios del síndrome de Kasabach-Merritt, que ocurre por activación plaquetaria en el interior de los grandes hemangiomas (3). Se observó, además, una ictericia preoperatoria con características hemolíticas, la cual se encuentra hasta en un $35 \%$ de los casos de hemangiomas hepáticos en niños (6).

Como alternativas en su tratamiento se incluye el uso de corticosteroides o agentes con un marcado efecto antiangiogénico (interferón alfa, ciclofosfamida, vincristina o actinomicina D) (9). Se ha descrito la terapia con bevacizumab, un anticuerpo monoclonal capaz de inhibir el factor de crecimiento endotelial; sin embargo, su eficacia no ha sido confirmada. Hay un pequeño número de casos en los que se considera la intervención quirúrgica por riesgo de complicaciones, que suelen ocurrir alrededor de la sexta semana de vida. El manejo quirúrgico incluye resecciones segmentarias, lobectomía o enucleación del hemangioma, mediante cirugía abierta o laparoscopia (10). Si se plantea la necesidad de cirugía, el procedimiento de elección es la enucleación (3). En este caso, la intervención quirúrgica fue la elección prioritaria, debido al compromiso ventilatorio secundario al efecto mecánico de la masa, que fue el doble del peso del hígado $(250 \mathrm{~g} v s .110 \mathrm{~g})$.

Es importante considerar como complicaciones durante el período postoperatorio a las hemorragias, las coagulopatías, las infecciones, las fístulas biliares, la ascitis y la falla hepática. A esta última se le atribuyó la presencia de la hiperbilirrubinemia directa de la paciente. Por otro lado, la CID podría considerarse como una complicación posquirúrgica debido a que, en el preoperatorio, la paciente no presentaba esta anomalía, lo que descarta que esta sea producto del síndrome de Kasabach-Merritt, que también suele presentarla. Las principales consecuencias de la CID son el consumo o la pérdida de las proteínas hemostáticas y de las plaquetas en el perioperatorio, que origina fenómenos hemorrágicos y obstrucciones trombóticas en la microcirculación, con compromiso de la función de diversos órganos. Específicamente, en este caso, una hemorragia pulmonar masiva.

\section{REFERENCIAS}

1. Chandrasoma P, Taylor C. Patología general. 2.a edición. México: Editorial Manual Moderno; 1998. p. 348. 
2. Gnarra M, Behr G, Kitajewski A, Wu JK, Anupindi SA, Shawber CJ, et al. History of the infantile hepatic hemangioma: From imaging to generating a differential diagnosis. World J Clin Pediatr. 2016;5(3):273-80. doi: 10.5409/wjcp. v5.i3.273.

3. Benavides C, García C, Rubilar P, Covachevich S, Perales C, Ricarte F, et al. Hemangiomas hepáticos. Rev Chil Cir. 2006;58(3):194-8. doi: 10.4067/S071840262006000300006.

4. Dabbs DJ. Diagnostic immunohistochemistry. 2.a edición. Filadelfia: Elsevier; 2006. p. 80-1.

5. Kuroda T, Hoshino K, Nosaka S, Shiota Y, Nakazawa A, Takimoto T. Critical hepatic hemangioma in infants: recent nationwide survey in Japan. Pediatr Int. 2014;56(3):304-8. doi: $10.1111 /$ ped.12347.
6. Costa CM, Rodrigues KE, Campos HG, Gibelli N, Ayoub A, Kayabashi M, et al. [Hepatic hemangioma]. J Pediatr. 2000;76(1):87-90. doi: 10.2223/JPED.39.

7. Sharma A, Kaspar M, Siddiqui M, Kim J. Enucleation after Embolization of Liver Failure-Causing Giant Liver Hemangioma. Am J Case Rep. 2015;24:563-7. doi: 10.12659/AJCR.893298.

8. Kumar V, Abbas A, Astra J. Robbins and Cotran pathologic basis of disease. 9.a edición. Filadelfia: Elsevier; 2015.p.862-3.

9. Cabrita SV, Gonçalves S, Rodrigues H, Guerra N, Moura P. Antenatal diagnosis of congenital hepatic hemangioma: a case report. Cases J. 2009;2:6829. doi: 10.4076/1757-16262-6829.

10. Bajenaru N, Balaban V, Săvulescu F, Campeanu I, Patrascu T. Hepatic hemangioma -review-. J Med Life. 2015;8 Spec Issue: 4-11. 